Article

\title{
Power Domain Multiplexing Waveform for 5G Wireless Networks
}

\author{
Korhan Cengiz ${ }^{1}$, Imran Baig ${ }^{2}$, Sumit Chakravarty ${ }^{3}$, Arun Kumar ${ }^{4}$, Mahmoud A. Albreem ${ }^{5}$, Mohammed \\ H. Alsharif ${ }^{6}$, Peerapong Uthansakul ${ }^{7, *}$, Jamel Nebhen ${ }^{8}$ and Ayman A. Aly ${ }^{9}$ \\ ${ }^{1}$ Department of Electrical-Electronics Engineering, Trakya University, 22030, Edirne, Turkey \\ ${ }^{2}$ Department of Electrical and Computer Engineering, College of Engineering, Dhofar University, Salalah 211, \\ Sultanate of Oman \\ ${ }^{3}$ Department of Electrical and Computer Engineering, Kennesaw State University, GA, USA \\ ${ }^{4}$ Department of ECE, JECRC University, Jaipur, 303905, India \\ ${ }^{5}$ Department of Electronics and Communication Engineering, A'Sharqiyah University, Ibra, 400, Oman \\ ${ }^{6}$ Department of Electrical Engineering, College of Electronics and Information Engineering, Sejong University, \\ Seoul, 05006, Korea \\ ${ }^{7}$ School of Telecommunication Engineering, Suranaree University of Technology, Nakhon, Ratchasima, Thailand \\ ${ }^{8}$ Prince Sattam bin Abdulaziz University, College of Computer Engineering and Sciences, Alkharj, 11942, Saudi Arabia \\ ${ }^{9}$ Department of Mechanical Engineering, College of Engineering, Taif University, Taif, 21944, Saudi Arabia \\ *Corresponding Author: Peerapong Uthansakul. Email: uthansakul@sut.ac.th \\ Received: 14 April 2021; Accepted: 15 May 2021
}

\begin{abstract}
Power domain non-orthogonal multiple access combined with a universal filtered multi-carrier (NOMA-UFMC) has the potential to cope with fifth generation $(5 \mathrm{G})$ unprecedented challenges. NOMA employs powerdomain multiplexing to support several users, whereas UFMC is robust to timing and frequency misalignments. Unfortunately, NOMA-UFMC waveform has a high peak-to-average power (PAPR) issue that creates a negative affect due to multicarrier modulations, rendering it is inefficient for the impending 5G mobile and wireless networks. Therefore, this article seeks to presents a discrete Hartley transform (DHT) pre-coding-based NOMA enabled universal filter multicarrier (UFMC) (DHT-NOMA-UFMC) waveform design for lowering the high PAPR. Additionally, DHT precoding also takes frequency advantage variations in the multipath wireless channel to get significant bit error rate (BER) gain. In the recommended arrangement, the throughput of the system is improved by multiplexing the users in the power domain and permitting the users with good and bad channel conditions to concurrently access the apportioned resources. The simulation outcomes divulge that the projected algorithm accomplished a gain of $5.8 \mathrm{~dB}$ as related to the conventional framework. Hence, it is established that the proposed DHT-NOMA-UFMC outperforms the existing NOMA-UFMC waveform. The key benefit of the proposed method over the other waveforms proposed for $5 \mathrm{G}$ is content gain due to the power domain multiplexing at the transmitting side. Thus, a huge count of mobile devices could be supported under specific restrictions. DHTNOMA-UFMC can be regarded as the most effective applications for $5 \mathrm{G}$
\end{abstract}




\begin{abstract}
Mobile and Wireless Networks. However, the main drawback of the proposed method is that the Fourier peak and phase signal is not easily estimated.
\end{abstract}

Keywords: NOMA-UFMC; 5G; PAPR; BER; DHT-NOMA-UFMC

\title{
1 Introduction
}

New forms of multiple and unique carrier waveforms have been introduced for the fifth generation $(5 \mathrm{G})$ cell radio networks. In $5 \mathrm{G}$ and beyond $5 \mathrm{G}(\mathrm{B} 5 \mathrm{G})$, data rates, capacity, lower latency and quality of service (QoS) are expected to be better than previous generations. Thus, this new era of technology is in the hands of $5 \mathrm{G}$ network systems, also known as new radio (NR) leading to a very smart and efficient method compared to conventional systems of wireless communications. The mobile industry that began in 1970 has seen many generations such as $1 \mathrm{G}-4 \mathrm{G}$. The introduction of $1 \mathrm{G}$ technology was in the form of analog signal transfers; later, digital methods is applied new second generation (2G) technology. When the demand increased from voice to data, $3 \mathrm{G}$ then evolved. However, to save the lost signal and information and provide better services known as quality service (QoS), an entirely new technology was introduced, known as fourth generation (4G) [1]. It is a well-known fact that approximately after the cycle of 10 years a new method of wireless communication systems developed to meet out the requirements and demands of consumers and provide them growth in digital devices across the globe. As per the trend, it is judged that digital data demands will scale up by about $30 \%$ in the next few years, thus achieving a high efficiency will be a great challenge for the next mobile network technology [2]. Wireless communications technology has an enormous capacity to change the way we work, behave and do the things we do every day. $5 \mathrm{G}$ is likely to restructure traditional services by providing better efficiency for work in industrial automation, the medical and health sector, and internet of things (IoT) devices. Although, in the path of $5 \mathrm{G}$, still there are many questions to answer and it's yet to provide a proper solution to all the issues it has to address. $5 \mathrm{G}$ is a technology capable of a realistic and upbeat impact on our lives. The $3^{\text {rd }}$ generation partnership Project (3GPP) indicates that, 5G mainly affects three kinds of applications: enhanced mobile broadband (EMB), device to device (D2D) communication and low latency [3]. Strict requirements that the aforementioned application families have are uninterrupted connectivity, huge system throughput, and enhanced spectral efficiency [4]. To satisfy the novel challenges in the 5G cellular radios and beyond, more novel multiple access and modulation schemes are being investigated, as the current waveforms are not able to fulfill the necessities and problems posed by the future $5 \mathrm{G}$ systems [5]. Recently, a large quantity of research has been performed to investigate the pertinence of Universal Filtered Multi-Carries (UFMC) for the 5G cellular radio nets [6]. Fig. 1a illustrates the conceptual view of UFMC waveform, where the whole spectrum is split into a couple of bands. All sub-bands have a particular couple of orthogonal carriers. To decrease Outof-Band Emissions (OBE) these sub-bands are filtered. The filtering besides reducing the filter span considerably when compared with the other $5 \mathrm{G}$ schemes. Additionally, sub-band filters provide design flexibility and reduce the Interference. Therefore, the UFMC is considered by some to be the best option for short burst transmission [7]. The UFMC shape also improves the spectral accuracy by not requiring any type of cyclic prefix (CP). NOMA employed a Super Coding (SC) and Successive Interference Cancellation (SIC) at the sending and receiving side of the transceiver. SC helps to access the allocated resources simultaneously by the numbers of sub-carriers. The separation of sub-carriers is accomplished in the power domain and SIC helps to decode the information of the particular users by treating any other signal as noise and finally cancelling it. In the projected framework, a cluster of filters are applied to the group of sub-carriers, which 
helps to maintain the signal separation, make the system compatible to cognitive radio (CR), and overcome the effect of frequency and time error. The NOMA UFMC is considered as an important contender for $5 \mathrm{G}$ radio due to several advantages, such as large device connectivity, efficient utilization of spectrum, high data speed, better connectivity, high capacity, and compatible to the advanced techniques [8]. Spectral ineffectiveness, however, is the foremost shortcoming of the typical UFMC, making it impracticable for tomorrow's 5G systems [9]. The UFMC therefore needs to be more rigorously studied and improved to address the problem of spectral inefficiency. Fig. 1a shows the basic structure of the NOMA-UFMC, where $k$ power range users can be grouped together to support a large number of mobile devices.

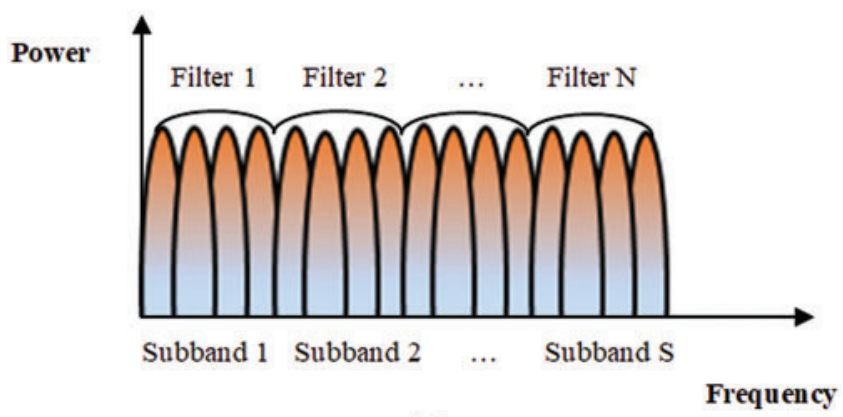

(a)

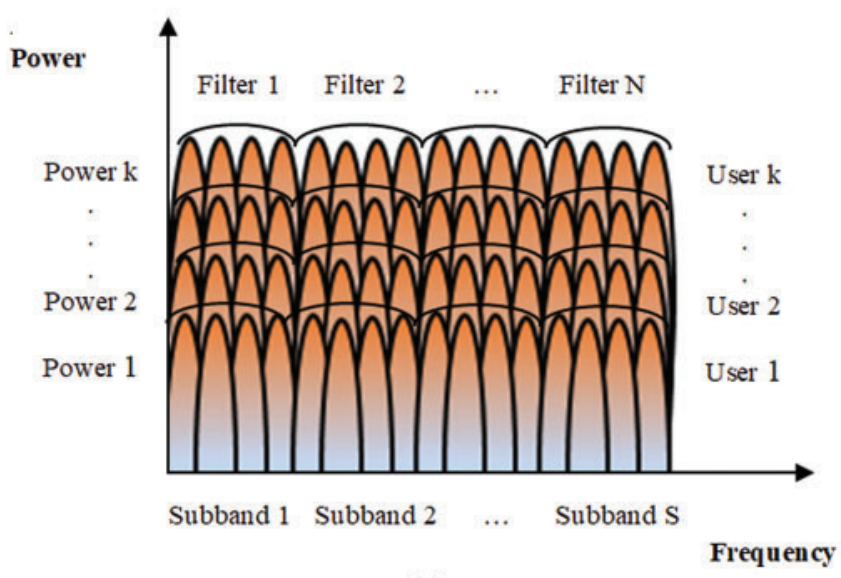

(b)

Figure 1: UFMC waveform. (a) UFMC waveform (b) NOMA-UFMC waveform

Fig. $1 \mathrm{~b}$ also illustrates that each user $k$ consumes an identical frequency band, but with different output levels to boost system capacity and performance. Unfortunately, NOMA-UFMC waveform suffers from high PAPR. The key objectives of the projected work are as follows:

- A significant reduction in peak power is accomplished in the hybrid waveform schemes. It is seen that by selecting the different value of $\mathrm{V}$ in the projected algorithm, the ideal peak power of the framework can be achieved.

- The spectral performance of the system is greatly enhanced by integrating the NOMA and UFMC waveforms thus making it suitable for $5 \mathrm{G}$ radio. 
- The interference is overcome by implementing SIC at the receiver of the framework and the resources are simultaneously accessed by the number of subscribers. Hence, the throughput of the projected framework is greatly enhanced.

The overall organization of the paper is: Section 1 summarizes related works; Section 2 compendiously explains the proposed waveform design. Section 3 evaluates the math formulas. Section 4 presents the results and Section 5 completes the work

\section{Related Work}

The DFT spread OFDM (DFT-s-OFDM) is common transmission radio implemented in 4G frameworks because of its low PAPR [10]. The DFT-s-OFDM inherits positive points from its forerunner CP-OFDM like outstanding spectrum performance. In CP-OFDM, the IDFT has greater modification that can be decreased by implementing DFT procedure, formerly the IDFT. Furthermore, the method of cyclic prefix (CP) in DFT-s-OFDM facilitates smooth treatment of multi path's impact. Moreover, it has very low deployment complexities, agile frequency allocation and strong MIMO adaptability. Yet, just like CP-OFDM, the DFT-S-OFDM plus sustains a loss high in $\mathrm{OBE}$ and cannot promote $5 \mathrm{G}$ requirements [11]. For tackling the issues mentioned above, a number of waveform designs were proposed in the state-of-the-art literature. Those are especially proposed to come through the new stiffness's of 5G cellular nets and beyond. Filter bank multicarrier (FBMC) [12], multi-carrier NOMA (MC-NOMA) [13] and universal filtered multicarrier (UFMC) [14] are included by those waveforms related to the recommended work. The UFMC is most likely introduced for $5 \mathrm{G}$ cellular networks. The UFMC comes all the benefits into FBMC and OFDM, in turn while keeping clear of their handicaps [15]. The waveform of NOMA, OFDM and UFMC is shown in Fig. 2. The debated methods ensure OBE as analogized $4 \mathrm{G}$ long term evolution advance (LTE-A) CP-OFDM. However, the spectral ineffectiveness is still the main problem of standard UFMC that makes it infeasible for upcoming $5 \mathrm{G}$ systems. The symbols and abbreviations used in the paper is given in Tab. 1.

\section{Proposed Waveform}

Fig. 3 shows the structure of the proposed DHT pre-coding-based uplink NOMA-UFMC (DHT-NOMA-UFMC) waveform design. According to the Fig. 3, all the $k$ mobile devices are interfixed to the Base Station (BS) concurrently and utilizing the same frequencies but, with the different powers. Assume there are $k$ users, where all users are utilizing a full band of $N$ subcarriers. The QAM modulated symbol $X_{l}$ of whichever $i^{\text {th }}$ subscriber, subsequently $\mathrm{S} / \mathrm{P}$ transformations is given as:

$X_{l}=\left[X_{0}, X_{1}, X_{2}, \ldots, X_{N-1}\right]^{T}$

$N$ corresponds to the count of subcarriers. Then QAM modulation process, the DHT precoding is performed to spread the modulated information and reduce the auto-correlation relationship amongst the modulated signal. S/P conversions, full-band $F$ of $N$ subcarriers for $i^{\text {th }}$ user is split into number bands for $C$ constant sub carries.

$x_{S_{i}}[n]=\frac{1}{\sqrt{C}} \sum_{l=0}^{C-1} X_{l} \cdot e^{j 2 \pi \frac{n}{C} l}, \quad 0 \leq n \leq C-1$ 
where $X_{l}$ shows the constellation symbols, contained in $S$ sub-bands. A cluster of filters of size $L$ is applied to the time domain (TD) signal of DHT-NOMA-UFMC, given as:-

$\breve{x}_{S_{i, l}}=x_{S_{i}} * f_{L}$

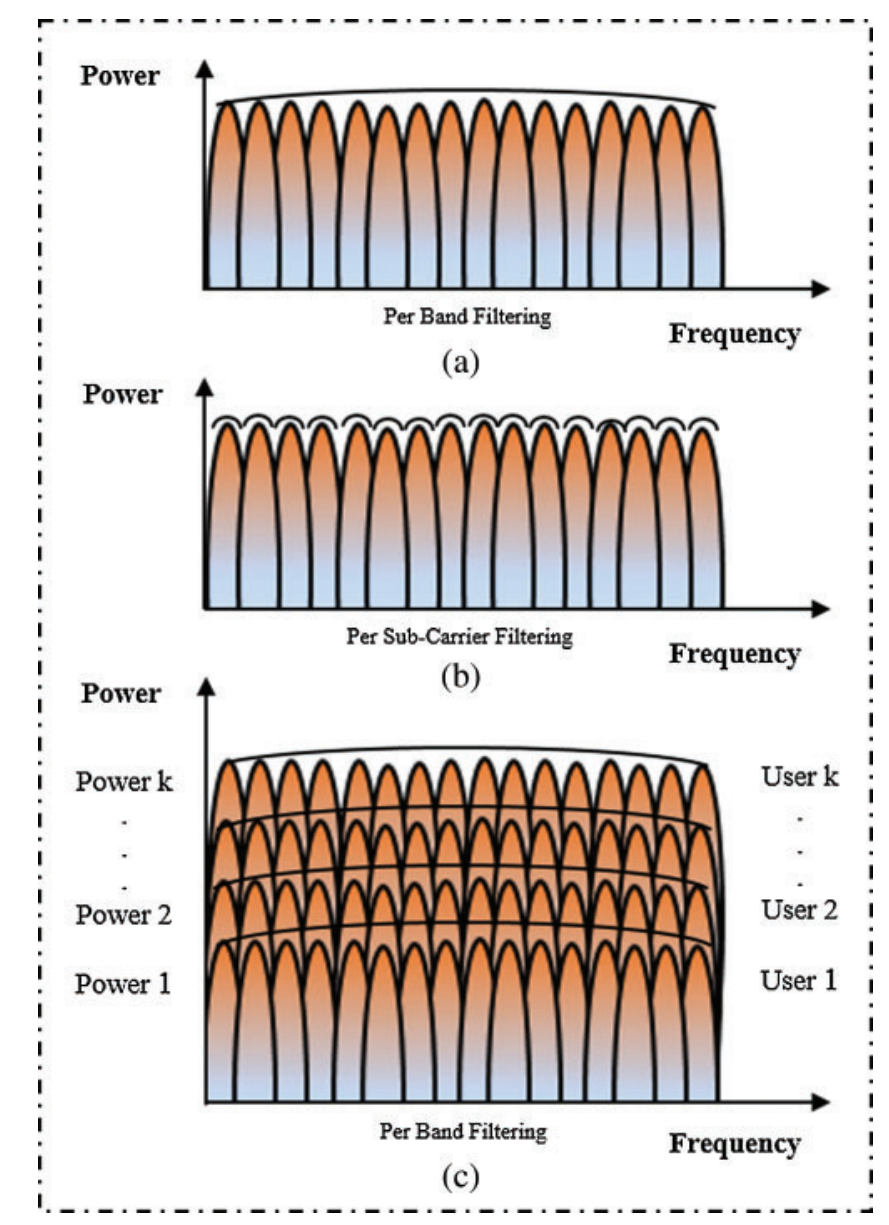

Figure 2: CP-OFDM, FBMC, and MC-NOMA waveforms comparisons

Once the filter procedures are carried out, the entire filtered sub-carriers are added to acquire the filtered signal, given as:

$x_{i}=\sum_{l=0}^{S-1} \breve{x}_{S_{i, l}}$ 
Table 1: Symbols definition

\begin{tabular}{ll}
\hline Symbol & Specification \\
\hline$X_{S}(n)$ & Modulated symbols \\
$f_{L}$ & Filter length \\
$\mathrm{C}$ & Constant sub-Carriers \\
$\mathrm{Y}$ & Received Signal \\
$\mathrm{N}$ & Number of sub-carriers \\
FFT & Fast Fourier Transform \\
TD & Time division \\
S/P & Serial to Parallel \\
QAM & Quadrature amplitude modulation \\
\hline
\end{tabular}

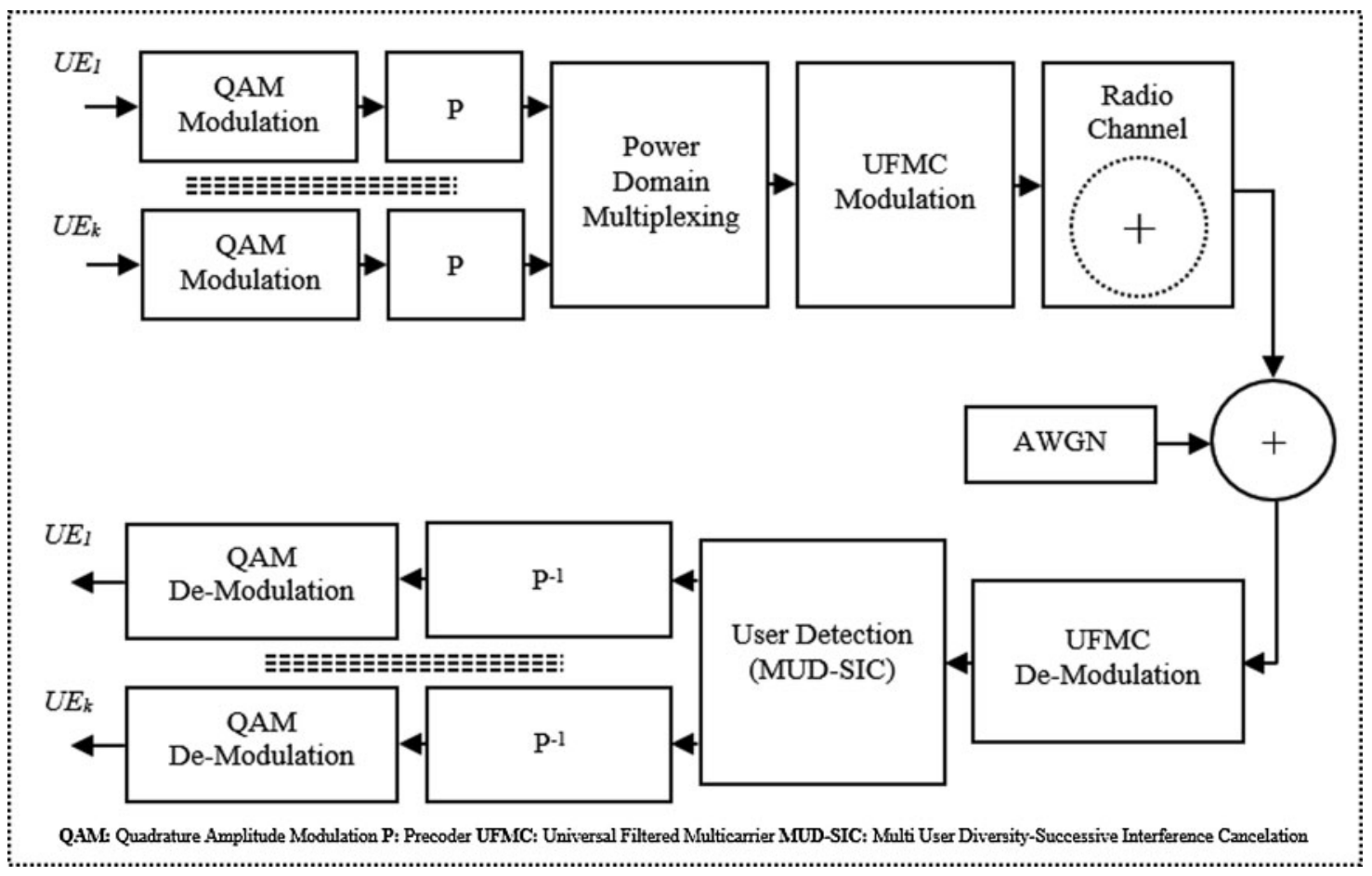

Figure 3: Proposed structure for downlink DHT-NOMA-UFMC waveform

where $x_{i}$ represent the UFMC symbol for $i^{\text {th }}$ user. Lastly, the NOMA-UFMC signal for $i^{\text {th }}$ user is multiplexed in the power domain, where several power ranks are chosen by the base station to each user disjoint. After the total power allocation $P_{t}$, all the transmitted signals are combined to get the DHT-NOMA-UFMC signal that can be given as follows:

$x=\sum_{i=1}^{k} \sqrt{p_{i}} x_{i}$ 
$p_{i}$ and $x_{i}$ represent allocated power and signals for any $i^{\text {th }} \mathrm{MU}$ respectively. The received signal is given as:

$y=\sum_{i=1}^{k} h_{i} \otimes x_{i}+w$

where $h_{i}$ is the time division (TD) channel coefficient for any $i^{\text {th }}$ MU. Per subcarrier equalization is applied to reduce the effects of network filters. Finally, the data is un-mapped to reconstruct the original signal.

Peak to average power ratio (PAPR) is the cause of worry in wireless communication and it is defined by the ratio of maximum power used in a transmitted sample symbol divided by the average power used in transmitting the whole symbol in OFDM networks.

The PAPR of the signal in Eq. (4) can be shown as:

$P A P R=\frac{\text { peak_power_of_x } x_{i}}{\text { average_power_of_x } x_{i}}$

The Complementary Cumulative Distribution Function (CCDF) is utilized to measure the PAPR. The CCDF of DHT-NOMA-UFMC signal is given as:

$P\left(P A P R>P A P R_{0}=1-\left(1-e^{-P A P R_{0}}\right)^{N}\right.$

PAPR0 shows the level of clipping.

\section{Numerical Results}

This section discusses the computer-based simulations and analyzes the proposed waveform in MATLAB. The PAPR performance has been investigated for the NOMA-UFMC, DHT-NOMAUFMC and Selective Mapping (SLM) based DHT-NOMA-UFMC waveforms, respectively. The data is randomly produced and several modulation schemes 4-QAM, 16-QAM and 64-QAM modulations are utilized. Tab. 2 presents various constraints utilized for evaluations and analyzes such constraints in detail. It also shows the simulation constraints of the projected work. In the projected work, we have taken the FFT equivalent to 512, sub-bands are 12 and sub-carriers are also equal to 20. The Dolph-Chebyshev filter is implemented with length $L=75$ with Stop-Band Attenuation 38.

Table 2: Simulation parameter

\begin{tabular}{ll}
\hline Parameter & Specification \\
\hline Size of FFT & 512 \\
Size of sub-band & 25 \\
Sub-bands & 12 \\
Filter & Dolph-Chebyshev \\
Sampling frequency & $15.36 \mathrm{MHz}$ \\
PAPR algorithm & DHT and SLM-DHT \\
Channel bandwidth & $10 \mathrm{MHz}$ \\
\hline
\end{tabular}


Figs. 4-6, present a simulation analysis of the PAPR for NOMA-UFMC, DHT-NOMAUFMC, DHT-NOMA-UFMC with SLM $(V=2)$ and DHT-NOMA-UFMC with SLM (V = 4) waveforms, respectively by using 4-QAM, 16-QAM and 64-QAM. It can be realized from Figs. 4-6 that the PAPR of the proposed DHT-NOMA-UFMC is efficient than the conventional NOMA-UFMC waveform. Furthermore, PAPR of the projected system can be further decreased by employing any kind of PAPR reduction scheme, which is suitable for the UFMC. We selected Selective-Mapping (SLM) and combined it with DHT precoding. Both SLM and DHT schemes are selected due to linearity, simplicity, maturity and their suitability for UFMC. It is also noticeable that DHT precoding or SLM or combination of both DHT precoding and SLM improves the PAPR gain. The PAPR gain of the proposed DHT-NOMA-UFMC waveform can be further improved by using the higher values in the $V$ of SLM thought the system complexity will increase. Therefore, selection of $V$ in SLM should be carefully selected, but this is another area of research beyond the scope of this study. The peak power performance of the projected system, after applying the reduction algorithm to 4-QAM is given in Fig. 4. At CCDF of $10^{-2}$, the recommended system accomplished a gain of $4,1.4$ and $0.6 \mathrm{~dB}$ as compared to other methods. It is also seen that by increasing the value of $V$, the peak power performance is greatly enhanced. However, the complexity of the system will be high for large value of V. Hence, it is important to find the finest PAPR value, without increasing the complexity.

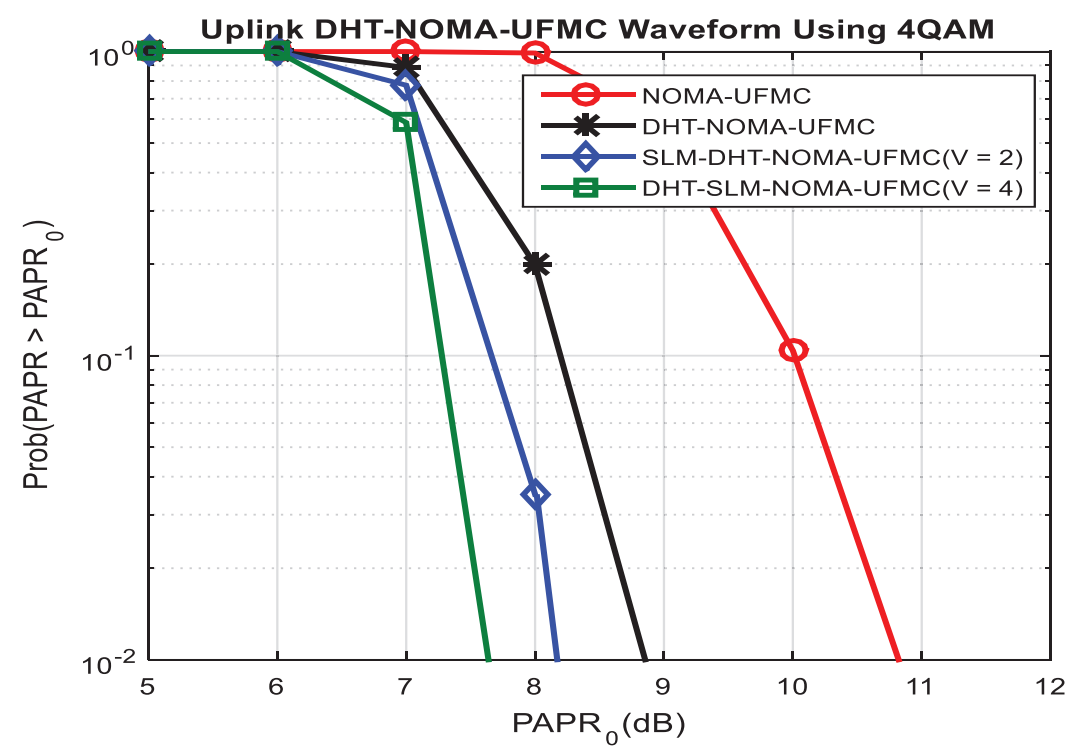

Figure 4: PAPR analyses by utilizing 4-QAM

In Fig. 5, the reduction algorithms are applied on NOMA-UFMC for a 16-QAM transmission method. In this case the interference will be more as compared to 4-QAM, since we are transmitting 4 symbols at a time. From the graph, it is noticed that the peak power performance of the 4-QAM is better than the 16-QAM. The projected system achieved a gain of 2.8, 1.6 and $0.3 \mathrm{~dB}$ as compared to different techniques. The best result is achieved at $V=4$. 


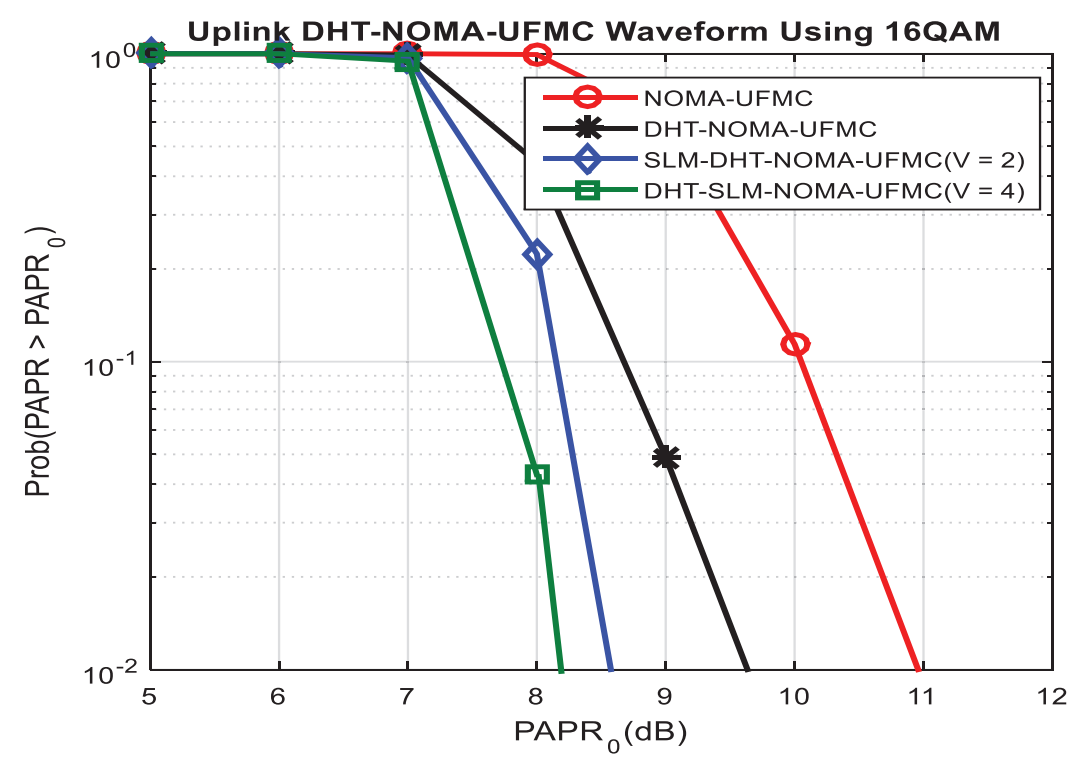

Figure 5: PAPR analyses by utilizing 16-QAM

The peak performance of the NOMA-UFMC for 64-QAM is given in the Fig. 6. In this case the interference will be high as compared to the 4-QAM and 16-QAM, since we are transmitting 6 symbols at a time. The utilization of 64-QAM will increase the capacity, spectral performance and data speed. However, there is a maximum probability that the peak power performance of the system will be low. From Fig. 6, it is seen that the peak power values of the reduction algorithm is large as compared to other transmission schemes. The peak power value obtained after applying a reduction method are $8.4 \mathrm{~dB}$ for DHT-SLM $(V=4), 8.8$ for DHT-SLM $(V=2)$ and $9.8 \mathrm{~dB}$ for DHT as equated to the peak power of NOMA-UFMC $11.2 \mathrm{~dB}$ (reference).

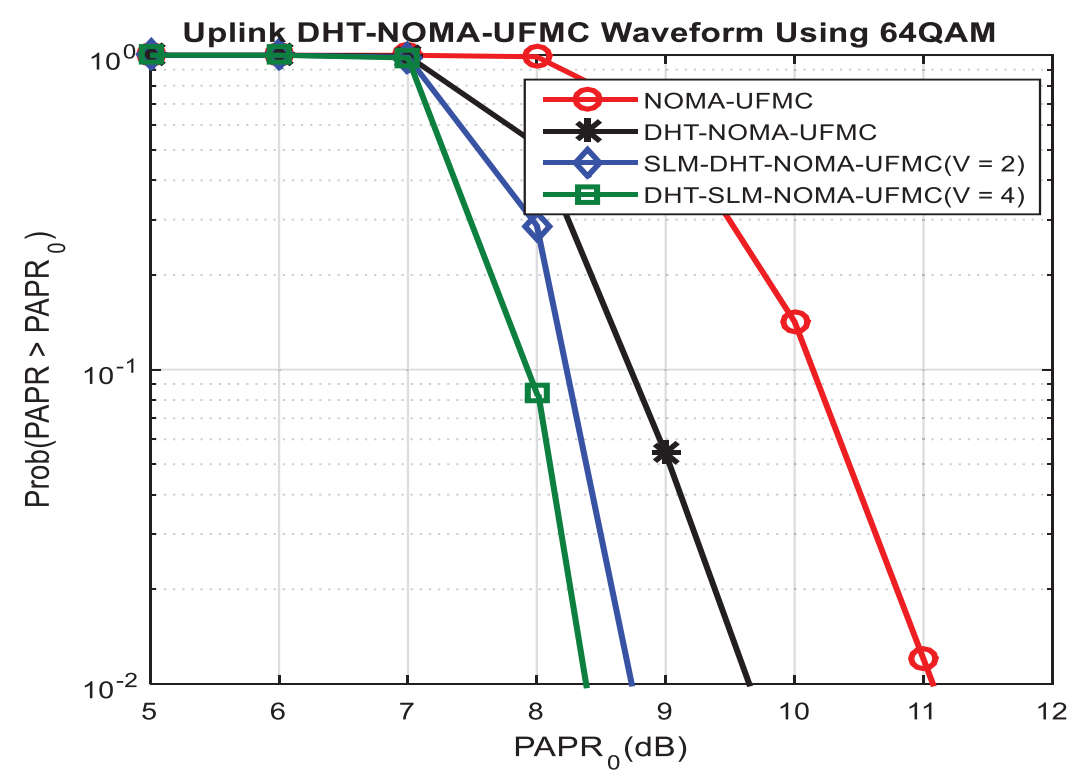

Figure 6: PAPR analyses by utilizing 64-QAM 
To analyze the throughput of the projected framework, the SNR Vs BER curves are given in Fig. 7. At the BER of $10^{-3}$, the SNR requirement of NOMA-UFMC is $8.8 \mathrm{~dB}$, DHTNOMA-UFMC is $7.4 \mathrm{~dB}$, SLM-NOMA-UFMC $(V=2)$ is $5.8 \mathrm{~dB}$ and SLM-NOMA-UFMC $(V=4)$ is $4.2 \mathrm{~dB}$. Hence, it is concluded that the projected framework is an improvement over the conventional methods.

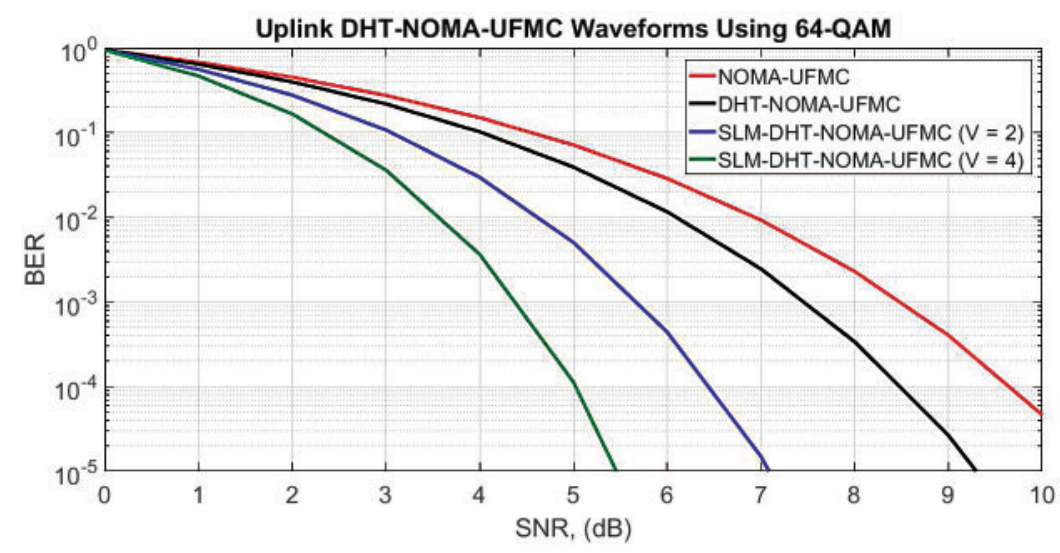

Figure 7: SNR vs. BER for 64-QAM

Fig. 8 indicated the throughput comparison of OFDM, UFMC, NOMA and NOMA-UFMC. It is seen that the projected method achieved a gain of 5.8, 4.2 and $2.8 \mathrm{~dB}$ as compared to the traditional waveform schemes.

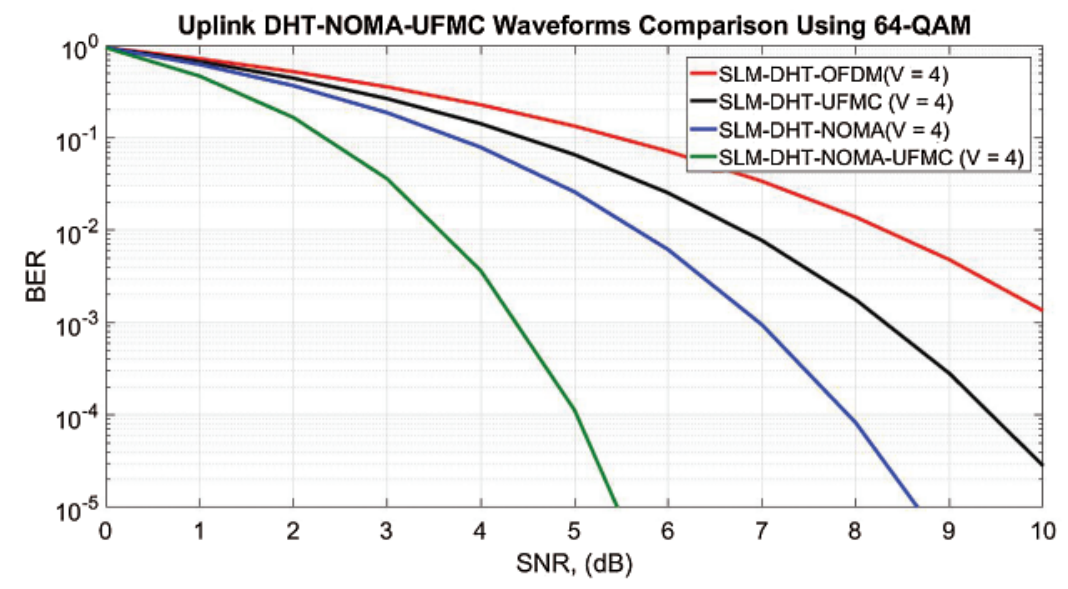

Figure 8: SNR vs. BER

Tab. 3 presents a summary of the PAPR performances of the different waveforms by using different QAMs. The effect of various QAMs is more apparent on the DHT Pre-coded NOMAUFMC waveforms and DHT Pre-coded NOMA-UFMC waveforms based on $\operatorname{SLM}(V=2,4)$. The PAPR performance degrades with higher modulation order. Hence, modulation order should be carefully selected. 
Table 3: PAPR comparison in $\mathrm{dBs}$

\begin{tabular}{llll}
\hline Waveforms & 4-QAM & 16-QAM & 64-QAM \\
\hline NOMA-UFMC & 10.8 & 10.9 & 11.1 \\
DHT-NOMA-UFMC & 8.8 & 9.6 & 9.7 \\
DHT-NOMA-UFMC using SLM $(V=2)$ & 8.2 & 8.6 & 8.7 \\
DHT-NOMA-UFMC using SLM $(V=4)$ & 7.6 & 8.2 & 8.4 \\
\hline
\end{tabular}

Tab. 4 Summarizes the performance analysis of the different 5G candidate waveforms. The complexity of the proposed waveform is directly proportional to the number of sub-bands and their lengths. The complexity can be decreased up to 4.5 times by utilizing poly-phase implementations. The complexity of the proposed wave form can also be decreased by implementing less length of FFTs and a lower number of sub bands [16]. Currently, a hardware design is implemented in [17] to decrease the complexity of UFMC based waveform.

Consequently, it could be concluded that the suggested DHT-NOMA-UFMC waveform could hold more users as compared with the existing UFMC by using power domain multiplexing. When the proposed waveform is compared with other $5 \mathrm{G}$ candidates, its advantages are content gain and throughput gain to support a great number of $5 \mathrm{G}$ users.

Table 4: Comparative analysis of the $5 \mathrm{G}$ candidate waveforms

\begin{tabular}{lll}
\hline Waveform & Advantages & Disadvantages \\
\hline SC-FDMA & 1. Low PAPR & 1. Low spectral efficiency \\
& 2. Low BER & 2. Low throughput \\
3. Low complexity & 3. High OOBE \\
4. Short-packet burst & 4. Low throughput \\
CP-OFDM & support & 1. High PAPR \\
& 1. Simple FDE & 2. Low spectral efficiency \\
2. Medium BER & 3. High OOBE \\
3. Low complexity & 4. No short-packet burst \\
FBMC & support \\
& 1. Medium spectral & 5. Low throughput \\
efficiency & 1. High PAPR \\
2. Low OOBE & 2. No short-packet burst \\
3. Medium BER & support \\
4. Medium throughput & 3. High complexity \\
1. High spectral efficiency & 1. High PAPR \\
2. High throughput & 2. High OOBE \\
& & 3. No short-packet burst \\
& & 4. High complexity
\end{tabular}


Table 4: Continued

\begin{tabular}{|c|c|c|}
\hline Waveform & Advantages & Disadvantages \\
\hline UFMC & $\begin{array}{l}\text { 1. Medium spectral } \\
\text { efficiency } \\
\text { 2. Short-packet burst } \\
\text { support } \\
\text { 3. Low OOBE } \\
\text { 4. Low BER } \\
\text { 5. Medium throughput } \\
\text { 6. Medium spectral } \\
\text { efficiency }\end{array}$ & $\begin{array}{l}\text { 1. High PAPR } \\
\text { 2. High complexity }\end{array}$ \\
\hline Proposed DHT-NOMA-UFMC & $\begin{array}{l}\text { 1. Higher spectral } \\
\text { efficiency } \\
\text { 2. Short-packet burst } \\
\text { support } \\
\text { 3. Low OOBE } \\
\text { 4. Low BER } \\
\text { 5. Low PAPR } \\
\text { 6. Higher throughput }\end{array}$ & 1. High complexity \\
\hline
\end{tabular}

\section{Conclusion}

In this study, we proposed and designed a new DHT-NOMA-UFMC for the future 5G mobile and wireless networks. The simulations in MATLAB were made to evaluate the PAPR performances. DHT pre-coding and SLM combined with DHT precoding is employed to reduce the PAPR. According to the evaluations and analyzes, the proposed waveform out-performs the standard NOMA-UFMC. The implementations complexities of the projected transmission techniques are very high, but they can be decreased by determining lower level FFTs and restricting the count of sub-bands. Further, the main drawback of the proposed method is that Fourier peak and phase signal is not easily estimated. The key benefit of the proposed method over the other waveforms proposed for $5 \mathrm{G}$ is content gain due to the power domain multiplexing at the transmitting side. Thus, a huge count of mobile devices could be supported under specific restrictions. DHT-NOMA-UFMC can be regarded as the most effective applications for 5G Mobile and Wireless Networks.

Funding Statement: This work was supported by SUT Research and Development Funds and by Thailand Science Research and Innovation (TSRI). Also, this work was supported by the Deanship of Scientific Research at Prince Sattam bin Abdulaziz University, Saudi Arabia. In addition, support by the Taif University Researchers Supporting Project number (TURSP-2020/77), Taif University, Taif, Saudi Arabia.

Conflicts of Interest: The authors declare that they have no conflicts of interest to report regarding the present study. 


\section{References}

[1] M. H. Alsharif, R. Nordin, M. Shakir and A. Ramly, "Small cells integration with the macro-cell under LTE cellular networks and potential extension for 5G," Journal of Electrical Engineering and Technology, vol. 14, no. 6, pp. 2455-2465, 2019.

[2] M. H. Alsharif, A. Kelechi, J. Kim and J. H. Kim, "Energy efficiency and coverage trade-off in 5G for eco-friendly and sustainable cellular networks," Symmetry, vol. 11, no. 3, pp. 408-428, 2019.

[3] A. Kumar, M. A. Albreem, M. H. Alsharif, A. Jahid, P. Uthansakul et al., "An efficient genetic hybrid papr technique for 5G waveforms," Computers, Materials and Continua, vol. 67, no. 3, pp. 3283-3292, 2021.

[4] P. Popovski, K. F. Trillingsgaard, O. Simeone and G. Durisi, "5G wireless network slicing for eMBB, URLLC, and mMTC: A communication-theoretic view," IEEE Access, vol. 6, no. 3, pp. 55765-55779, 2018.

[5] Y. Liu, Z. Qin, M. Elkashlan, Z. Ding, A. Nallanathan et al., "Non orthogonal multiple access for 5G and beyond," Proc. of the IEEE, vol. 105, no. 12, pp. 2347-2381, 2017.

[6] A. Kumar, M. Gupta, D. Le and A. A. Aly, "PTS-papr reduction technique for 5G advanced waveforms using BFO algorithm," Intelligent Automation and Soft Computing, vol. 27, no. 3, pp. 713-722, 2021.

[7] M. H. Alsharif and R. Nordin, "Evolution towards fifth generation (5G) wireless networks: Current trends and challenges in the deployment of millimetre wave, massive MIMO, and small cells," Telecommunication Systems, vol. 64, no. 4, pp. 617-637, 2017.

[8] A. Kumar and M. Gupta, "A comprehensive study of PAPR reduction techniques: Design of DSLMCT joint reduction technique for advanced waveform," Soft Computing, vol. 24, no. 2, pp. 11893-11907, 2020.

[9] M. H. Alsharif, A. H. Kelechi, M. A. Albreem, S. Chaudhry, M. Zia et al., "Sixth generation (6G) wireless networks: Vision, research activities, challenges and potential solutions," Symmetry, vol. 12, no. 4, pp. 26-35, 2020.

[10] I. Baig, U. Farooq, M. Zghaibeh, N. Hasan and A. Sajid, "A Power domain UFMC system design for underwater optical communication with reduced PAPR," Journal of Internet Technology, vol. 22, no. 2, pp. 395-401, 2021.

[11] H. G. Myung, J. Lim and D. J. Goodman, "Single carrier FDMA for uplink wireless transmission," IEEE Vehicular Technology Magazine, vol. 1, no. 3, pp. 30-38, 2006.

[12] F. Boroujeny, "OFDM vs. filter bank multicarrier," IEEE Signal Processing Magazine, vol. 28, no. 3, pp. 92-112, 2011.

[13] Z. Ding, X. Lei, G. K. Karagiannidis, R. Schober, J. Yuan et al., "A survey on non-orthogonal multiple access for 5G networks: Research challenges and future trends," IEEE Journal on Selected Areas in Communications, vol. 35, no. 10, pp. 2181-2195, 2017.

[14] J. Doré, R. Gerzaguet, N. Cassiau and D. Ktenas, "Waveform contenders for 5G: Description, analysis and comparison," Physical Communication, vol. 24, no. 2, pp. 46-61, 2017.

[15] I. Almeida, L. Mendes, J. Rodrigues and M. da Cruz, "5G waveforms for IoT applications," IEEE Communications Surveys \& Tutorials, vol. 21, no. 3, pp. 2554- 2567, 2019.

[16] A. Kumar and H. Rathore, "Modified DSLM technique for PAPR reduction in FBMC system," Radioelectronics and Communication System, vol. 62, no. 4, pp. 416-421, 2019.

[17] A. R. Jafri, J. Majid, M. A. Shami, M. A. Imran and M. N. U. Islam, "Hardware complexity reduction in universal filtered multicarrier transmitter implementation," IEEE Access, vol. 5, no. 3, pp. 1340113408, 2017. 